\title{
CALCIFIED URACHAL REMNANT MIMICKING AS BLADDER WALL CALCIFICATION - CASE SERIES
}

Parthasarathi $\mathrm{A}^{1}$, Gautham $\mathrm{M}^{2}$, Pravin G $\mathrm{U}^{3}$

HOW TO CITE THIS ARTICLE:

Parthasarathi A, Gautham M, Pravin G.U. "Calcified Urachal Remnant Mimicking as Bladder Wall Calcification Case Series". Journal of Evolution of Medical and Dental Sciences 2014; Vol. 3, Issue 02, January 13; Page: 272 278, DOI:10.14260/jemds/2014/1821

ABSTRACT: Computer tomography of kidneys, ureters and bladder (CT KUB) is the main investigation in suspected renal tract calculi. Ultrasound of kidneys, ureters and bladder (KUB) region can come in hand during acute conditions especially in patients with ureteric calculus causing hydronephrosis and hydroureter. However, several pathologies other than renal tract calculi can cause apparent urinary bladder calcification. We describe series of cases who presented with renal colic. CT KUB performed on admission revealed a calcified urachal remnant mimicking a urinary bladder wall calcification, confirmed by reviewing the multi- planar reformatted images. We also discuss the differential diagnoses that should be considered when presented with urinary bladder calcification. Our study shows urachus calcification is much more common in patients than previously taught and more common in older patients of more than 50 years than younger patients. Males are commonly affected than females.

KEY WORDS: Urachus, Calculus, Urachal remnant, hydronephrosis, hydroureter, Computed tomography.

INTRODUCTION: The urachus or median umbilical ligament is a midline tubular structure that extends upward from the anterior dome of the bladder toward the umbilicus. It is a vestigial remnant of at least two embryonic structures: the cloaca, which is the cephalic extension of the urogenital sinus (a precursor of the fetal bladder), and the allantois, which is a derivative of the yolk $\operatorname{sac}^{1,2}$. The tubular urachus normally involutes before birth, remaining as a fibrous band with no known function. However, persistence of an embryonic urachal remnant can give rise to various clinical problems, not only in infants and children but also in adults. Because urachal remnant diseases are uncommon and manifest with nonspecific abdominal or urinary signs and symptoms, definitive presurgical diagnosis is not easily made. Various abnormalities can be confusing unless one is familiar with the basic embryologic anatomy and imaging features of the subumbilical and prevesical region. Because computed tomography (CT) and ultrasonography (US) display crosssectional images and the urachus in the anterior abdominal wall is located away from interfering intestinal structures, these modalities are ideally suited for demonstrating urachal anomalies ${ }^{2-6}$. Calcification of urachal remnant is very rare entity and we present a series of cases in this article.

CLASSIFACTION OF URACHAL ANOMALIES: Urachal anomalies are due to failure of complete obliteration of the lumen during gestation ${ }^{7,8}$. Their anatomical classification is based on the degree to which the patency of the urachus has persisted. Typical urachal anomalies are shown in Figure 1. The possibilities vary from a completely patent urachus that allows urine to freely drain through the umbilicus to a small blind-ending sinus tract from the skin. An urachal cyst may be present at any location along the length of the urachus but are most commonly found near the dome of the bladder. 
An urachal diverticulum is a partial patency of the urachus draining into the dome of the bladder. The anomalies shown in Figure 1.

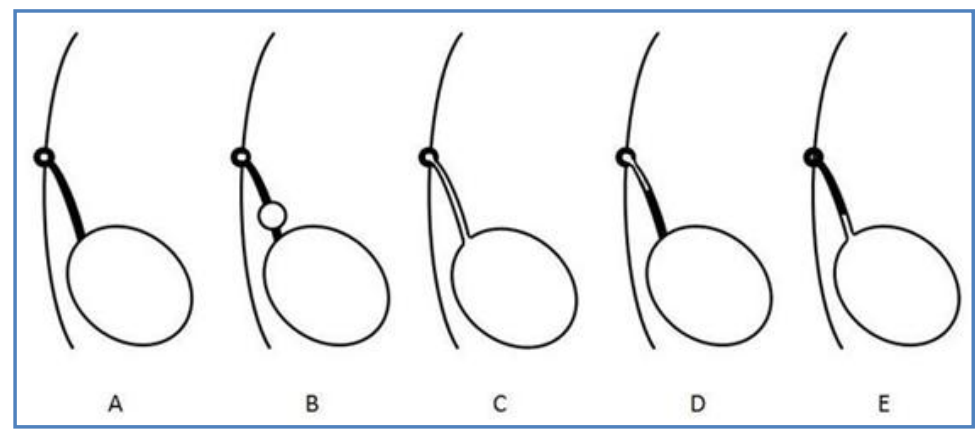

FIG: 1 CLASSIFACTION OF URACHAL ANOMALIES

A- Normal obliteration of the Urachal lumen. B- Urachal cyst. C- Patent urachus. D- Urachal sinus. E- Urachal diverticulum.

MATERIALS AND METHODS: This study was performed from January 2013 to November 2013 in the department of Radio-diagnosis, Rajarajeswari Medical College and hospital, Bengaluru, Karnataka on 600 patients presenting with renal colic and other urinary tract symptoms.

INCLUSION CRITERIA: Study involves the patients more than 30 years presenting with renal colic and lower abdominal pain, microscopic hematuria, macroscopic hematuria and recurrent urinary tract infections.

CT PROTOCOLS: Non-enhanced and contrast enhanced CT (whenever possible) was performed on 600 patients from domes of diaphragm to pubic symphysis employing $5 \mathrm{~mm}$ thick slices in supine position. Oral contrast was avoided in all the cases in view of contrast interfering in the detection of the calculus. All the images are viewed in soft tissue window and bone window. Multi- planar reformatted imaging done whenever necessary.

DISCUSSION: We report case series of 8 cases showing incidental calcified urachalremnant mimicking a bladder wall calcification diagnosed in patients with renalcolic and lower abdominal pain from CT KUB (Fig. 2 to Fig. 9). The presence of calculi with in a vesicour achaldiverticulum has only been described once before, where the calcific densities were clearly demonstrated with in a diverticulum distinct from the urinary bladder wall9,10.This is the first case series to describe calcification within a urachal remnant so close to its insertion into the urinary bladder wall that mimicsa bladder wall calcification /calculuson CT imaging.

The urachus, or median umbilical ligament, is a midline tubular structure that extends upward from the anterior dome of the bladder toward the umbilicus. It is a vestigial remnant of at least two embryonic structures: the cloaca, which is the cephalic extension of the urogenital sinus (a precursor of the fetal bladder), and the allantois, which is a derivative of the yolk sac ${ }^{1,2}$. The tubular urachus normally involutes before birth, remaining as a fibrous band with no known function. Occasionally, the urachus may persist and result in a variety of clinical problems. Such 
urachalanomaliesoccurat an incidence of 1 in 5000 births, being two-fold more common in men than women ${ }^{10}$. They usually manifest in childhood. There are no known risk factors, but they may occur in association with other congenital renaltract anomalies. Four congenital urachalanomalie scan occur, including patent urachus, urachalcyst, umbilical-urachal sinus, vesicourachal diverticulum. In the absence of concomitant infection, most patients with urachalanomalies are asymptomatic. When symptomatic, they can be treated by surgical excision. Prognosisis good as they are usually benign 10

In our cases the patients presenting with renal colic and lower abdominal pain, CTKUB has replaced the abdominal radiograph as the first line investigation for patients presenting with renalcolic ${ }^{11}$. However, conventional radiography may have arolein monitoring radio-opaque renal calculi during treatment with lithotripsy. An ultrasound of the renaltract may be performed to assess for hydronephrosis or hydroureter if there is the suspicion of an obstructed urinary system which would warrant decompression. Other radiological investing at ions suchas C Turography are indicated in the investigation of haematuria to exclude upper tracturo thelialmalignancy in elderly patients but are not the initial investigation of choice for renalcolic. MRI is promising modality in patients where there is increased serum creatinine level due to urinary tract obstruction.

CTKUB most of the does not involve the administration of intravenous contrast medium. Some argue it should be performed with the patient in the prone position to facilitate differentiation between calculi impacted within the vesicoureteric junction from calculi free within the bladder ${ }^{12}$ Our patients were imaged both in supine and prone position. The calcification was demonstrated onaxialimages to be in the anterior bladder wall. This findings can be mistaken for a calculus lying within the dependent portion of the bladder had the axial images only from the prone CT been interrogated. Bone windows helps identify calcific calculi and should be routine lyper formed when interpreting a CTKUB when measuring the dimensions of calculi which have implications for patient management, e.g. conservative or surgery. It will also provide opportunity to exclude any bone lesion in the imaged skeleton. MPR images are routine lyat radiologists hand an should be reviewed in addition with standard axial. Maximum intensity projection images can be useful, especially in the coronal plane, to help identify the ureters, particularly in the distal portion which may be difficult to appreciate on axial images alone due to lack of intra- abdominal fator normal peristalsis ${ }^{10}$. In our case series MIP images revealed the calcific density located at the linear soft tissue structure extending from the antero superior aspect of the bladder, consistent with aurachal remnant. The calcified remnant was not large enough to demonstrate on conventional radiography and produce acoustic shadow on ultra sonographic scan. A contrast enhanced CT was not indicated but performed in few cases to rule out any other cause for pain but not showed enhancement of the area of interest. MR appearances of a calcified urachal remnant have not previously been described.

However, other pathological entities should be considered. Bladder calculi can form denovo, aphenomenon associated with urinary stasis from bladder outlet obstruction ${ }^{13}$. In this scenario the calculi will be free to move within the bladder and will adopt of dependent position on CT KUB. The CT may also demonstrate features of bladder outlet obstruction. Bladder calculi may be detect able on plain radiographs if of sufficient size and density. Calcificcalculi may cast acoustic shadows on ultrasound. They do not demonstrate enhancement following the administration of contrast.

Primary bladder tumors are important causes of bladder calcification, most commonly transitional cellcarcinoma ${ }^{13}$. CT is not the first line investigation for primary bladder malignancy, but if the bladder is distended CT can demonstrate focal bladder wall thickening. In older male patients, 
the prostate can calcify, enlarge and indent the bladder, giving the impression of posterior bladder wall calcification ${ }^{13}$. In the appropriate patient demographic, schistosomiasis should be considered as a cause of urinary bladder calcification. It is the most frequent cause of bladder wall calcification worldwide. However, this calcification is usually arcuate and associated with calcification in other areas of the urinary tract ${ }^{14}$. Another infection that can result in urinary bladder calcification is tuberculosis. Calcification of the upper renaltract is usually observed prior to spread to the distalureters and bladder ${ }^{15}$. Inflammation within the bladder can proceed to calcification. This has been documented in cyclophosphamide-induced cystitis ${ }^{13}$. Amyloidosisis another inflammatory condition that has been associated with urinary bladder calcification, albeitrarely ${ }^{16}$

Urachal carcinoma is a rare pathology. Calcification within such tumors has previously been described $^{17}$. If large enough, a calcified urachal carcinoma may be detectable as a calcific entity on plain film and may demonstrate acoustic shadowing on ultrasound. The presence of enhancing abnormal surrounding soft tissue on CT would help raise the suspicion. As the urachusis related to the anterior dome of the bladder, a calcified urachalcar cinoma may appear as wall calcification adjacent to the anterior bladder wall. However, calcification of urachal remnantatits insertion into the bladder wall, mimicking a bladder calculus has been demonstrated in our case series.

CONCLUSION: In our study we found 8 patients with urachal calcification withina urachal remnant so close to its insertion into the urinary bladder wall that mimics a bladder wall calcification /calculuson CT imaging. According to our study the urachus calcification is seen in about $1.33 \%$ that is about 1 in 75 patients. Out of 8 patients, 7 patients are of above 50 years and 1 patient is of 35years, this shows that urachal calcification is more common in older age group than the patients of young age. Out of 8 patients with urachus calcification 2 female and 6 male patients which shows urachus calcification is common in males than females.

\section{REFERENCES:}

1. Moore KL. The urogenital system. In: Moore KL, eds. The developing human. $3^{\text {rd }}$ ed. Philadelphia, Pa: Saunders, 1982; 255-297

2. Jeong-Sik Yu, MD, Ki Whang Kim, MD, Hwa-Jin Lee, MD, Young-Jun Lee, MD, Choon-Sik Yoon, MD, and Myung-Joon Kim, MD. Urachal Remnant Diseases: Spectrum of CT and US Findings. Radio Graphics 2001; 21:451-461

3. Avni EF, Matos C, Diard F, Schulman CC. Midline omphalovesical anomalies in children: contribution of ultrasound imaging. UrolRadiol1988; 10:189-194.

4. Boothroyd AE, Cudmore RE. Ultrasound of the discharging umbilicus. PediatrRadiol 1996; 26:362-364.

5. Cilento BG, Jr, Bauer SB, Retik AB, and Peters CA, Atala A. Urachal anomalies: defining the best diagnostic modality. Urology 1998; 52:120-122.

6. Khati NJ, Enquist EG, Javitt MC. Imaging of the umbilicus and periumbilical region. RadioGraphics 1998; 18:413-431.

7. MacNeily AE, Koleilat N, Kirulata HG, Homsey YL. Urachal abcesses: protean manifestations, thier recognition, and management. Urol 1992: 40:530-535

8. J. Christopher Austin, MD. Urachal AnomoliesIn Children. Pediatric urology book. 
9. Ozbulbul NI, Dagli M, Akdogan G, Olcer T. C Turography of a vesicour achal diverti culum containing calculi. Diagn Interv Radiol.2010Mar;16(1):56-8.PMID:19838994.

10. Jonathan Carl Luis Rodrigues, Sanjay Gandhi. Don't get caught out! A rare case of a calcified urachal remnant mimicking a bladder calculus. Journal of Radiology Case Reports. 2013Mar;7(3):34-38

11. Kennish SJ, Bhatnagar P, Wah TM, Bush S, Irving HC. Is the KUB radiograph redundant for investigating acute ureteric colic in the non-contrast enhanced computed tomography era? Clin Radiol2008 Oct;63(10):1131-5. PMID:18774360.

12. Freeman SJ, Sells H. Investigation of loinpain. Imaging 2005Aug;17(1):19-33.PMID:53048188.

13. Dyer R, Chen MYM, Zagoria RJ. Abnormal calcifications intheurinarytract.RadioGraphics1998Nov-Dec;18(6): 1405-24.PMID:9821191.

14. Fataar S, Rudwan M, Bassiony $H$, Satyanath S. CT of genitourinary calcification due to schistosomiasis. AustralasRadiol.1990 Aug;34(3):234-7.PMID:2125826.

15. Wang L J, Wu C F, Wong Y C, Chuang CK, Chu S H, Chen CJ. Imaging findings of urinary tuberculosis on excretory urographyandcomputerizedtomography.JUrol.2003Feb; 169(2):524-8.PMID:12544301.

16. Thomas SD, SandersPW3rd, Pollack H. Primary amyloidosis of urinary bladder and ureter: cause of mural calcification. Urology 1977 May; 9 (5): 586-9. PMID:871049.

17. Thali-Schwab CM, Woodward PJ, Wagner BJ. Computed tomographic appearance of urachal adenocarcinomas: reviewof25 cases.EurRadiol2005Jan;15(1):79-84. PMID:15258826.

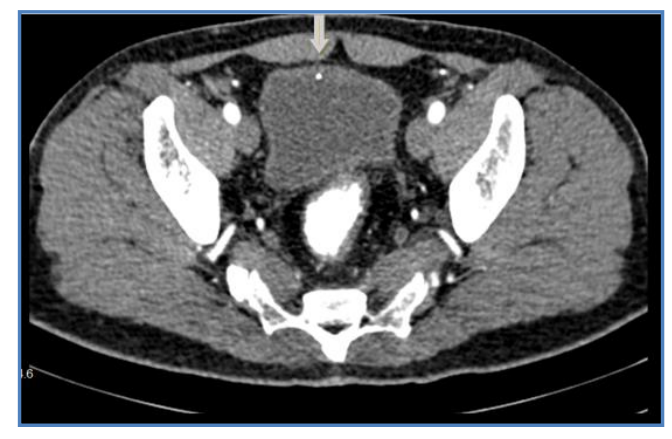

FIG 2: AXIAL CECT SHOWING URACHAL REMNANT CALCIFACTION IN 65 YEARS OLD MALE PATIENT

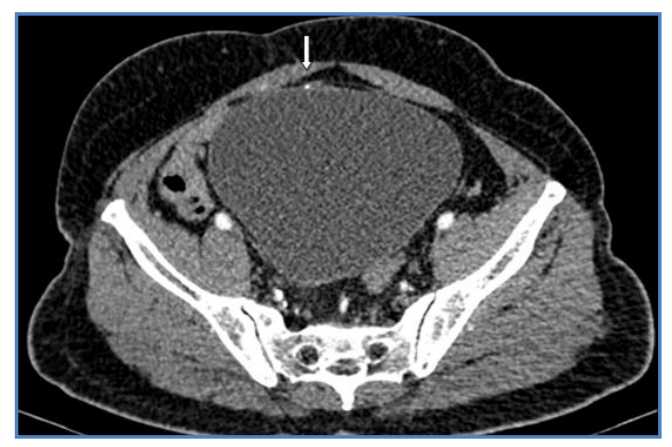

FIG 3: AXIAL CECT SHOWING URACHAL REMNANT CALCIFACTION IN 63 YEARS OLD MALE PATIENT 


\section{ORIGINAL ARTICLE}

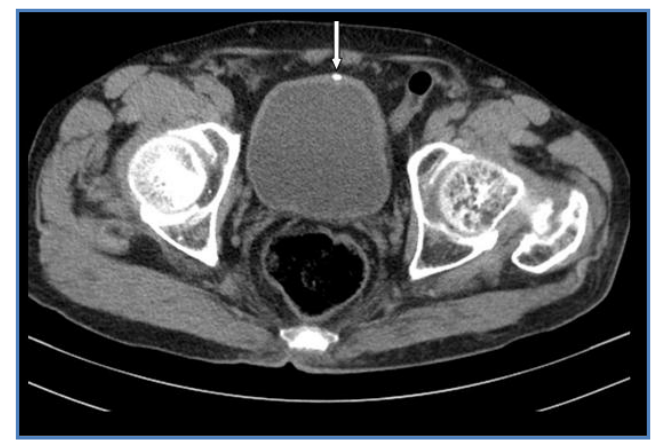

FIG 4: AXIAL NECT SHOWING URACHAL REMNANT

CALCIFACTION IN 6OYEARS OLD MALE PATIENT

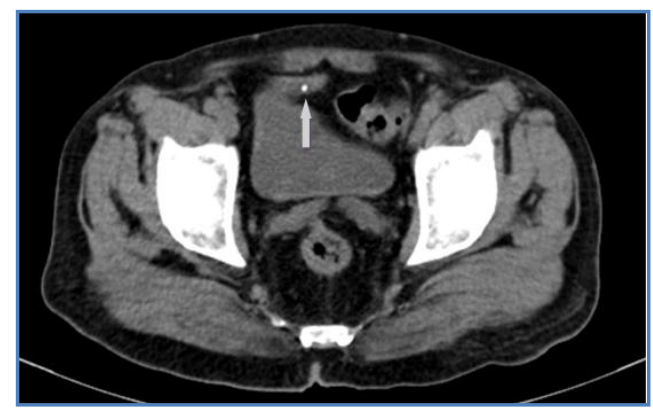

FIG 5: AXIAL NECT SHOWING URACHAL REMNANT CALCIFACTION IN 75YEARS OLD MALE PATIENT

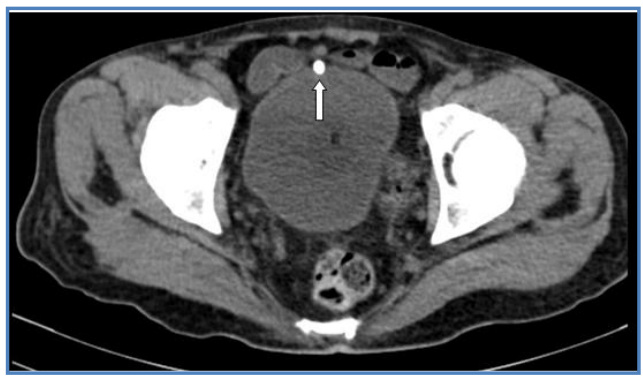

FIG 6: AXIAL NECT SHOWING URACHAL REMNANT CALCIFACTION IN 65 YEARS OLD MALE PATIENT

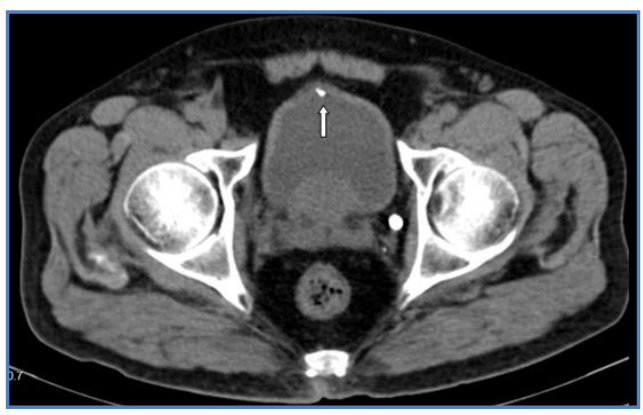

FIG 7: AXIAL NECT SHOWING URACHAL REMNANT CALCIFACTION IN 67 YEARS OLD MALE PATIENT

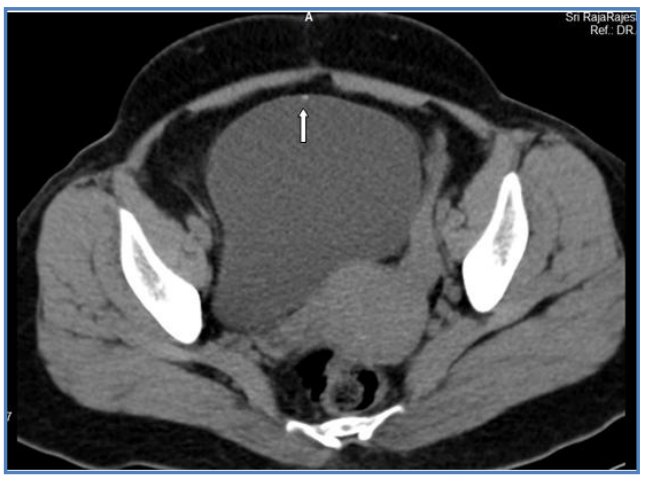

FIG 8: AXIAL NECT SHOWING URACHAL REMNANT CALCIFACTION IN 75 YEARS OLD FEMALE PATIENT 


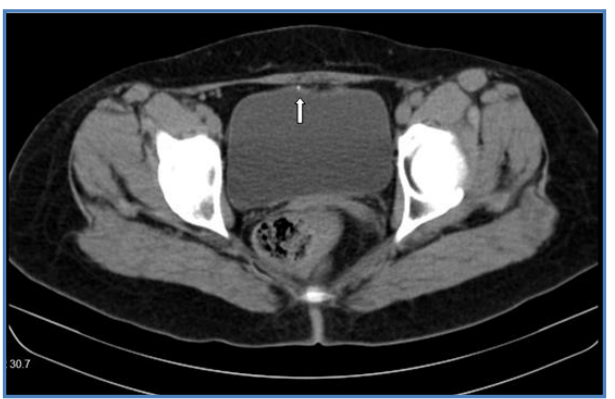

FIG 9: AXIAL NECT SHOWING URACHAL REMNANT

CALCIFACTION IN 50 YEARS OLD FEMALE PATIENT

\section{AUTHORS:}

1. Parthasarathi $\mathrm{A}$

2. Gautham M

3. Pravin G.U.

\section{PARTICULARS OF CONTRIBUTORS:}

1. Assistant Professor, Department of Radiology Uroradiology, Rajarajeswari Medical College and Hospital, Mysore Road, Bengaluru.

2. Assistant Professor, Department of Radiology Uroradiology, Rajarajeswari Medical College and Hospital, Mysore Road, Bengaluru.

3. Professor, Department of Radiology Uroradiology, Rajarajeswari Medical College and Hospital, Mysore Road, Bengaluru.

\section{NAME ADDRESS EMAIL ID OF THE CORRESPONDING AUTHOR:}

Dr. Parthasarathi A, Assistant Professor, Department of Radiology, Rajarajeswari Medical College, Mysore Road, Bangalore.

Email-dr.pathu@gmail.com

Date of Submission: 09/12/2013.

Date of Peer Review: 11/12/2013.

Date of Acceptance: 20/12/2013.

Date of Publishing: 08/01/2014 